Apidologie, 1977, 8 (1) 25-32.

\title{
MORPHOLOGISCHER UND ANATOMISCHER BAU DER EXTRAFLORALEN NEKTARIEN BEI DER SÜBKIRSCHE
}

\author{
Structure morphologique et anatomique des nectaires \\ extrafloraux de la cerise douce \\ Zofia KOLASA \\ Institut für Naturwissenschaftliche Grundlagen der Pflanzen- \\ produktion der Landwirtschaftlichen Akademie in Lublin*.
}

\section{SUMMARY}

\author{
MORPHOLOGIC AND ANATOMIC STRUCTURES \\ OF THE EXTRAFLORAL NECTARIES OF THE \\ SWEET CHERRY
}

The anatomic and morphologic structure of the extrafloral nectaries of the sweet cherry were investigated. The varieties " Hellrosa" and "Hedelfinger giant cherry" have the largest laminae, whereas the wild sweet cherry is equipped with the largest extrafloral nectaries. A correlation between the size of the lamina and the surface of the extrafloral nectaries could be established with all investigated varieties of the sweet cherry. No differences were found in the anatomic structure of the extrafloral nectaries. They represent a rarely occurring type of nectaries.

\section{ZUSAMMENFASSUNG}

Untersucht wurde der anatomische und morphologische Bau der extrafloralen Nektarien der Süßkirsche. Die größten Blattspreiten besitzen die Sorten Hellrosa und Hedelfinger Riesenkirsche. Die größten extrafloralen Nektarien kommen bei der Wildsüßkirsche vor. Festgestellt wurde eine Korrelation zwischen der Größe der Blattspreite und der Oberfläche der extrafloralen Nektarien bei allen untersuchten Sorten der Süßkirsche. Unterschiede im anatomischen Bau der extrafloralen Nektarien wurden nicht gefunden. Sie stellen einen selten vertretenen Nektarientyp dar.

* Die Arbeit wurde unter Leitung von Frau Prof. Dr. Zofia Demianowicz ausgeführt. 


\section{EINFÜHRUNG}

Die Süßkirsche (Cerasus avium L.) besitzt zwei Arten von Nektarien : florale Nektarien, die sich am vertieften Blütenboden befinden, und extraflorale Nektarien, die an den Blattstielen in der Nähe der Blattspreite angeordnet sind. Der Nektarienbau wurde von Frei (1955) untersucht, die feststellen konnte, daß die Nektarien aus kleinzelligem, tief in das zellenreiche Parenchym einwachsendem Sekretionsgewebe gebildet sind. Es wurde dabei von FreI darauf hingewiesen, daß kein Leitbündel zum Nektarium führt. Der Bau der extrafloralen Nektarien wurde von Böнmer (zitiert nach KuLiJEv 1959) beschrieben, wobei jedoch kein Leitgewebe unterschieden wurde.

Die extrafloralen Nektarien scheiden kleine Nektarmengen aus, die sehr schnell an der offenen Oberfläche dieser Drüse eintrocknen. Von Huszcza (1971) wurde die Nektarsekretion der beiden Nektarienarten bei vier Sorten der Süßkirsche untersucht, und zwar bei der Hellrosa, der Hedelfinger Riesenkirsche, der Frühesten der Mark und bei der Wildsüßkirsche. Er stellte fest, daß unter den extrafloralen Nektarien die der Wildsüßkirsche die reichlichste Nektarsekretion aufweisen und daß deren Nektar den höchsten prozentualen Zuckergehalt hat.

Nach KuliJev hängt die Menge des ausgesonderten Nektars von der Größe der Nektarien ab, unabhängig von deren Lage (1952). Frei-WyssLing und Agthe (1950) sind der Ansicht, daß die Zuckerkonzentration des Nektars eng verbunden ist mit der Beschaffenheit des Leitgewebes, das Wasser und Assimilate zuführt. Besteht dieses ausschließlich aus Phloem, so wird Nektar mit hohem Zuckergehalt abgesondert; dominieren dagegen Xylemelemente, so ist der Nektar wasserreich.

Das Ziel der vorliegenden Arbeit ist, die Größe der extrafloralen Nektarien und ihren anatomischen Bau bei vier Sorten der Süßkirsche zu untersuchen.

\section{MATERIAL UND METHODE}

Die morphologischen und anatomischen Untersuchungen der extrafloralen Nektarien bei der Süßkirsche wurden im Zeitraum 1971-1973 durchgeführt. Das Untersuchungsmaterial stammte aus der Obstanlage in Osiny bei Trzciniec. Diese Obstplantage wurde im Jahre 1950 auf Boden von podsoliertem Lösstypus angelegt.

Je 50 Laubblätter wurden von drei Bäumen jeder Süßkirschensorte abgerissen und danach die Oberfläche der Blattspreiten und die der extrafloralen Nektarien im Laufe von drei Jahren bei 150 Laubblättern jeder Sorte gemessen.

Die Oberfäche der Blattspreiten wurde gemessen, indem die Blätter auf ein Blatt Millimeterpapier abgezeichnet und die dabei ermittelte Größe in $\mathrm{cm}^{2}$ ausgedrückr wurde. Zur Messung der oberen Fläche der extrafloralen Nektarien wurde eine Binokularlupe mit einer in das Okular eingebauten Skala verwendet. Die Nektarien-Oberfläche nähert sich in ihrer Form 
einem Rechteck, aus diesem Grunde wurden sowohl ihre Länge als auch ihre Breite gemessen. Fanden sich am Blattstiel mehrere Nektarien, so wurde deren Gesamtoberfläche berücksichtigt. Auf Grund der gewonnenen Ergebnisse wurde für jedes Jahr getrennt der Korrelationskoeffizient zwischen der Größe der Oberfläche der extrafloralen Nektarien und der Größe der Blattspreite für jede der vier untersuchten Sorten ermittelt.

Nach den durchgeführten Untersuchungen wurden die Blattstiele samt Nektarien in 50-\% igem Athylalkohol mit Zugabe von Glyzerol fixiert. Aus diesem konservierten Material wurden mit Hilfe des Paraffinverfahrens und des Schlittenmikrotoms anatomische Präparate angefertigt und in Kanadabalsam eingebettet.

\section{BESPRECHUNG DER ERGEBNISSE}

Aus den Messungen der Oberfläche der Blattspreiten und der extrafloralen Nektarien der vier Sorten der Süßkirsche ist ersichtlich, daß die Süßkirschen-Sorten Hellrosa und Hedelfinger Riesenkirsche die größten Laubblätter besitzen. Für die Früheste der Mark und die Wildsüßkirsche sind kleinere Blattspreiten kennzeichnend, die sich hinsichtlich der Größe unwesentlich voneinander unterscheiden (Tabelle 1 und 3). Die größten extrafloralen Nektarien wurden bei der Wildsüßkirsche, die kleinsten bei der Frühesten der Mark festgestellt (Tabelle 2 und 3).

TАВ. 1. - Zahl der Blattspreiten in den einzelnen Größenbereichen.

TABL. 1. - Nombre de feuilles par classes de taille.

\begin{tabular}{|c|c|c|c|c|c|c|c|c|}
\hline \multirow{2}{*}{$\begin{array}{c}\text { Sorte } \\
\text { Variété }\end{array}$} & \multicolumn{8}{|c|}{$\begin{array}{l}\text { Oberfläche der Blattspreite in } \mathrm{cm}^{2} \\
\text { Surface des feuilles en } \mathrm{cm}^{2}\end{array}$} \\
\hline & $10-20$ & $20-30$ & $30-40$ & $40-50$ & $50-60$ & $60-70$ & $70-80$ & $80-90$ \\
\hline Hellrosa . . . . . . . . . . . . & 3 & 11 & 24 & 30 & 20 & 25 & 23 & 14 \\
\hline Hedelfinger Riesenkirsche......... & -- & 7 & 22 & 33 & 40 & 33 & 12 & 3 \\
\hline Frïheste der Mark . . . . . . . . & 23 & 30 & 31 & 19 & 17 & 19 & 11 & - \\
\hline Wildsüßkirsche $\ldots \ldots \ldots \ldots \ldots \ldots$ & 7 & 15 & 51 & 34 & 17 & 14 & 7 & 5 \\
\hline
\end{tabular}

TAB. 2. -- Zahl der Nektarien in den einzelnen Größenbereichen.

TABL. 2. - Nombre de nectaires par classes de taille.

\begin{tabular}{|c|c|c|c|c|c|c|c|c|c|c|c|}
\hline \multirow{2}{*}{$\begin{array}{l}\text { Sorte } \\
\text { Variété }\end{array}$} & \multicolumn{11}{|c|}{$\begin{array}{c}\text { Oberfläche der Nektarien in } \mathrm{cm}^{2} \\
\text { Surface des nectaires en } \mathrm{cm}^{2}\end{array}$} \\
\hline & 01 & $01-02$ & $02-03$ & 03-04 & $04-05$ & $05-06$ & $06-07$ & $07-08$ & $08-09$ & $09-10$ & 10 \\
\hline Hellrosa & 5 & 47 & 52 & 23 & 16 & 6 & 1 & - & - & - & 一 \\
\hline $\begin{array}{l}\text { Hedelfinger } \\
\text { Riesenkirsche. }\end{array}$ & 14 & 42 & 36 & 35 & 12 & 7 & 3 & 1 & - & - & - \\
\hline Früheste der Mark. & 38 & 52 & 31 & 16 & 9 & 4 & 一 & - & - & - & - \\
\hline Wildsü $\beta$ kirsche $\ldots$ & - & 4 & 13 & 23 & 22 & 28 & 15 & 18 & 12 & 5 & 10 \\
\hline
\end{tabular}


TaB. 3. - Mittlere Größe der Blattspreiten und der extrafloralen Nektarien bei den vier Sorten der Süßkirsche in $\mathrm{cm}^{2}$

TABL. 3. - Taille moyenne des feuilles et des nectaires exirafloraux chez les quatre variétés de cerise douce en $\mathrm{cm}^{2}$

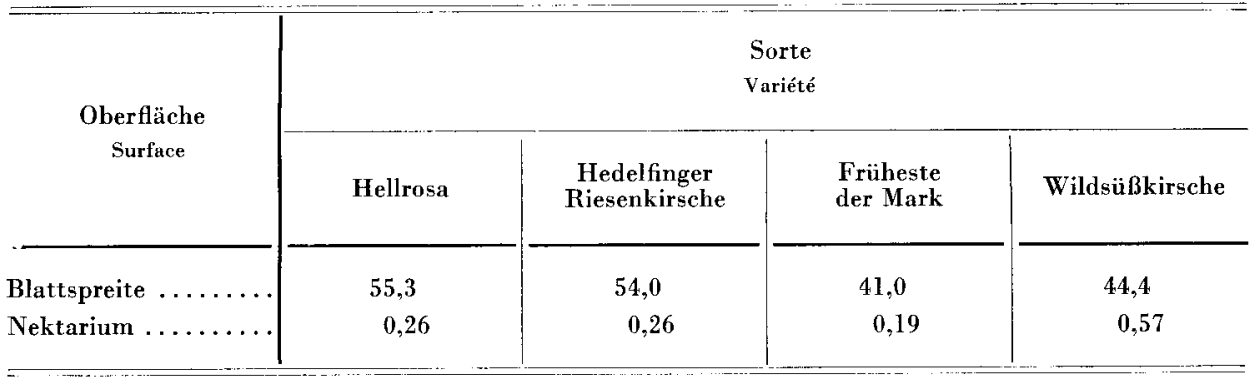

Tab. 4. - Werte des Korrelationskoeffizienten zwischen der Oberfläche der Blattspreite und der des extrafloralen Nektariums.

TABL. 4. - Valeurs des coefficients de corrélation entre la surface des feuilles et celle des nectaires extrafloraux.

\begin{tabular}{|c|c|c|c|c|}
\hline \multirow{2}{*}{$\begin{array}{l}\text { Versuchsjahr } \\
\text { Année }\end{array}$} & \multicolumn{4}{|c|}{$\begin{array}{l}\text { Sorte } \\
\text { Variété }\end{array}$} \\
\hline & Hellrosa & $\begin{array}{c}\text { Hedelfinger } \\
\text { Riesenkirsehe }\end{array}$ & $\begin{array}{l}\text { Früheste } \\
\text { der Mark }\end{array}$ & Wildsüßkirsche \\
\hline 1971 & 0,4 & 0,48 & 0,48 & $-0,1$ \\
\hline 1972 & $-0, \mathrm{I}$ & 0,4 & 0,2 & 0,7 \\
\hline 1973 & 0,4 & 0,6 & 0,5 & 0,8 \\
\hline
\end{tabular}

Zwischen der Größe der Blattspreite und der extrafloralen Nektarien besteht bei der Süßkirsche eine positive Korrelation (Tabelle 4). Bei den Sorten Hellrosa und Früheste der Mark kann sie als schwach, bei der Hedelfinger Riesenkirsche als mittelgroß bezeichnet werden. Die Wildsüßkirsche weist dagegen eine hohe positive Korrelation auf.

Dazu ist zu bemerken, daß die Werte der Korrelationskoeffizienten in neun Fällen statistisch gesichert sind. Statistisch nicht gesichert sind sie bei den Sorten Hellrosa und Früheste der Mark im Jahre 1972 und bei der Wildsüßkirsche im Jahre 1971.

Im anatomischen Bau der extrafloralen Nektarien der untersuchten Sorten der Süßkirsche wurden keine Unterschiede festgestellt. Kennzeichnend für die Nektarien ist eine 1-2 schichtige Oberhaut, deren längliche Zellen von 
einer dicken Kutikula überzogen sind (Abb. 1). Diese Oberhaut besitzt keine Spaltöffnungen. Der ausgeschiedene Nektar sammelt sich unter der Kutikula, die aufreißen kann, wenn sich größere Nektarmengen ansammeln. Unter der Epidermis liegt das Sekretionsgewebe, das aus Zellen mit zähflüssigem Plasma besteht, die kleiner sind als die Parenchymzellen, die den übrigen Teil des Nektariums ausfüllen.

Das extraflorale Nektarium wird durch ein Leitbündel mit Wasser versorgt. Dieses Leitbündel ist an der Basis des Nektariums dünn und bildet, nachdem es ins Nektariumgewebe eingedrungen ist, fächerförmige Verzweigungen (Abb. 2). Es ist zu bemerken, daß in vielen Parenchymzellen Drusen von Kalziumoxalat vorkommen.

Eingegangen im November 1976.

Reçu pour publication en novembre 1976.

\section{DANK}

Ich fühle mich verpflichtet, Frau Professor Dr. Z. Demianowicz an dieser Stelle meinen besten Dank auszusprechen. Sie hat mich auf den Fragenkreis hingewiesen, dem das Thema meiner Untersuchung entnommen wurde, und mich in meiner Arbeit freundlich unterstützt.

\section{RÉSUME}

On a étudié durant 3 ans, de 1971 à 1973, la structure morphologique et anatomique des nectaires extrafloraux chez 4 variétés de cerises douces (Cerasus avium L.) : les variétés « Heelrosa » et “ Hedelfinger » de la cerise géante, la variété “ Früheste der Mark » de la cerise douce et la variété sauvage de la cerise douce. On a choisi 3 arbres de chaque variété et prélevé sur chacun d'eux 50 feuilles. Puis on a mesuré la surface des feuilles et celle des nectaires. On a donc obtenu 150 mesures pour chaque variété.

On a tracé le contour des feuilles sur une feuille de papier millimétré, puis exprimé en $\mathbf{c m}^{2}$ la surface ainsi obtenue. Pour mesurer la surface des nectaires extrafloraux on a utilisé une loupe binoculaire à échelle incorporée. La forme des nectaires se rapprochant de celle d'un rectangle, on en a mesuré la longueur et la largeur. S'il existait plusieurs nectaires sur le pétiole de la feuille, on prenait en compte leur surface totale. On a calculé le coefficient de corrélation entre la taille des feuilles et la surface des nectaires extrafloraux pour chacune des 4 variétés étudiées et pour chaque année.

Pour réaliser les préparations anatomiques des nectaires extrafloraux on a paraffiné le matériel, fixé préalablement à l'alcool à $50 \%$ additionné de glycérol, et on a fait des coupes au microtome.

Il ressort de ces mesures que les variétés de cerises géantes " Hellrosa » et " Hedelfinger " possèdent les feuilles les plus grandes. La variété ( Früheste der Mark ) de la cerise douce et la cerise douce sauvage ont toutes deux des feuilles plus petites (tabl. 1 et 3 ). Les plus grands nectaires extrafloraux se rencontrent chez la cerise douce sauvage, les plus petits chez la variété “ Früheste der Mark » (tabl. 2 et 3). Il existe chez la cerise douce une corrélation positive entre la taille des feuilles et celle des nectaires extrafloraux (tabl. 4). 


\section{PLANCHE I}

\section{PLATE I}

Авв. 1. - Fragment eines extrafloralen Nektariums der Süßkirsche (Cerasus avium $L$ ) mit der von dicker Kutikula überzogenen Epidermis (Längsschnitt).

Fig. 1. - Fragment d'un nectaire extrafloral de la cerise douce (Cerasus avium L.) montrant l'épiderme recouvert d'une épaisse cuticule (coupe longitudinale).

(Foto Janusz Kolasa)

Авв. 2. -- Längsschnitt des extrafloralen Nektariums der Süßkirsche.

Fig. 2. - Coupe longitudinale du nectaire extrafloral de la cerise douce.

(Foto Janusz Kolasa) 

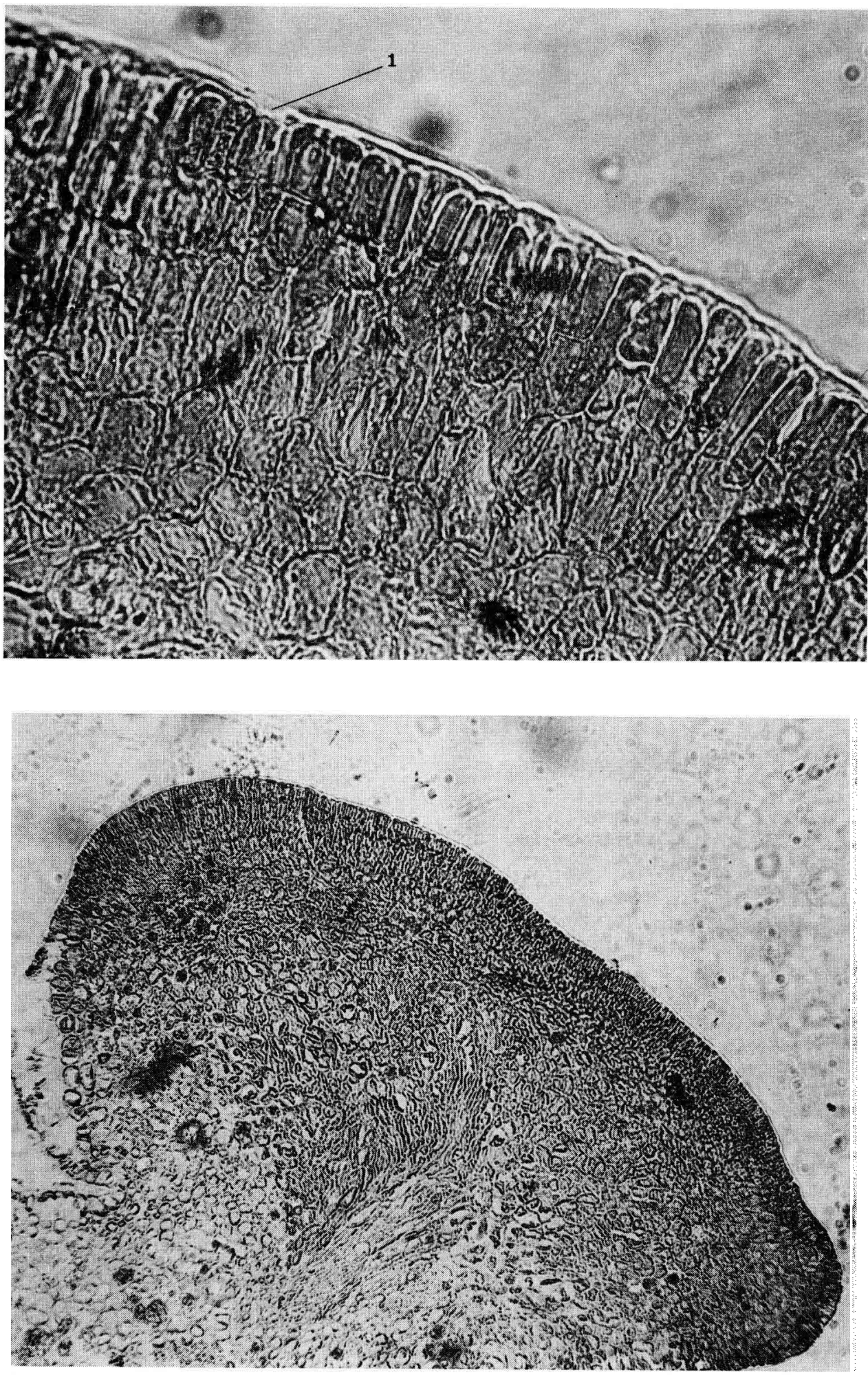
On n'a pu mettre en évidence aucune différence dans la structure anatomique des nectaires extrafloraux des variétés étudiées. Ces nectaires se caractérisent par un épiderme simple ou double, dont les cellules longitudinales sont revêtues d'une épaisse cuticule (fig. 1). Dans cet épiderme il n'y a pas de stomates. Sous l'épiderme on trouve le tissu secréteur, composé de cellules plus petites que les cellules du parenchyme qui constituent le reste du nectaire. A la base du nectaire le faisceau vasculaire, qui sort du pédoncule, est fin; puis il dessine des bifurcations en éventail, après avoir pénétré dans le tissu du nectaire.

\section{LITERAT URVERZEICHNIS}

Frei E., 1955, Die Innervierung der floralen Nektarien dikotyler Pflanzenfamilien. Berichte der Schweizerischen Botanischen Gesellschaft. 65: 60-114.

Frey-Wessling A., Agthe C., 1950, Nektar ist ausgeschiedener Phloemsaft. Verh. Schweiz. Naturforsch. Ges. 130, Vers. Davos. 175-176.

HuszczA W., 1972, Biologia kwitnienia, nektarowanie i wydajnosc miodowa czeresni. Maschinenschrift, Landwirtschaftliche Akademie in Lublin.

Kulijev A. M., 1952, Zadaczi izuczenija miedonosnych i pierganosnych rastieni j. Moskau.

KuliJEv A. M., 1959, Morfologiczeskaja ewoljucija nektarnikow pokrytosemjannych rastenij. Kirowabad. 